\title{
Calcium Ion Exchange in Crystalline Gelsolin
}

\section{Sakesit Chumnarnsilpa ${ }^{1,2} \dagger$, Anantasak Loonchanta ${ }^{1} \dagger$, Bo Xue ${ }^{1,2}$ Han $\mathrm{Choe}^{3}$, Dunja Urosev ${ }^{4}$, Hui Wang ${ }^{4}$, Uno Lindberg ${ }^{5}$ Leslie D. Burtnick ${ }^{4 *}$ and Robert C. Robinson ${ }^{1,2}$}

\author{
${ }^{1}$ Department of Medical \\ Biochemistry and Microbiology \\ Uppsala Biomedical Center \\ Uppsala University, Uppsala \\ 751 23, Sweden \\ ${ }^{2}$ Institute of Molecular and Cell \\ Biology, 61 Biopolis Drive \\ Proteos, Singapore 138673 \\ ${ }^{3}$ Department of Physiology \\ University of Ulsan College of \\ Medicine, Songpagoo \\ Poongnapdong 388-1, Seoul \\ 138-736, South Korea \\ ${ }^{4}$ Department of Chemistry and \\ Centre for Blood Research \\ The University of British \\ Columbia, Vancouver, BC \\ Canada V6T $1 Z 1$ \\ ${ }^{5}$ Department of Cell Biology \\ Wenner-Gren Institute \\ Stockholm University, 10691 \\ Stockholm, Sweden
}

${ }^{*}$ Corresponding author
Gelsolin is a calcium and pH-sensitive modulator of actin filament length. Here, we use X-ray crystallography to examine the extraction and exchange of calcium ions from their binding sites in different crystalline forms of the activated $\mathrm{N}$ and C-terminal halves of gelsolin, G1-G3 and G4-G6, respectively. We demonstrate that the combination of calcium and low $\mathrm{pH}$ activating conditions do not induce conformational changes in G4-G6 beyond those elicited by calcium alone. EGTA is able to remove calcium ions bound to the type I and type II metal ion-binding sites in G4-G6. Constrained by crystal contacts and stabilized by interdomain interaction surfaces, the gross structure of calcium-depleted G4-G6 remains that of the activated form. However, high-resolution details of changes in the ionbinding sites may represent the initial steps toward restoration of the arrangement of domains found in the calcium-free inactive form of gelsolin in solution. Furthermore, bathing crystals with the trivalent calcium ion mimic, $\mathrm{Tb}^{3+}$, results in anomalous scattering data that permit unequivocal localization of terbium ions in each of the proposed type I and type II ionbinding sites of both halves of gelsolin. In contrast to predictions based on solution studies, we find that no calcium ion is immune to exchange.

(C) 2006 Elsevier Ltd. All rights reserved.

Keywords: gelsolin; actin; calcium activation; protein crystallography; conformational change

\section{Introduction}

Cell movement and platelet activation are examples of processes in which actin filament numbers, positions and lengths are controlled dynamically by severing, capping and uncapping mechanisms that involve gelsolin. ${ }^{1-3}$ During platelet activation, transient increases in phosphatidylinositol 4,5-bisphosphate $\left(\mathrm{PIP}_{2}\right)$ hydrolysis and calcium ion release result in gelsolin activation and the severing of actin filaments. These events

$\dagger$ S.C. and A.L. contributed equally to this work.

Abbreviations used: G1, G2, G3, G4, G5, and G6, the domains of gelsolin from the $\mathrm{N}$ to the $\mathrm{C}$ terminus; $\mathrm{G} 1-\mathrm{G} 3$, $\mathrm{N}$-terminal half of gesolin; G4-G6, C-terminal half of gelsolin; $\mathrm{PIP}_{2}$, phosphatidylinositol 4,5-bisphosphate.

E-mail address of the corresponding author: burtnick@chem.ubc.ca are closely followed by $\mathrm{PIP}_{2}$ accumulation in the membrane, which leads to the removal of the gelsolin caps and massive polymerization of actin at the large number of newly exposed filament ends. ${ }^{2}$

The two halves of the gelsolin are held in an inactive state by interaction of G6 and the C-terminal extension with G2 (tail latch). Activation at physiological $\mathrm{pH}$ involves calcium, or calcium in conjunction with actin, releasing the tail latch and revealing the major actin-binding sites on G1, G2-G3 and G4. ${ }^{4-6}$ The structural basis of $\mathrm{pH}$ activation of gelsolin below pH 6.0 is unknown. ${ }^{7}$ G1-G3 and G4-G6 are biologically relevant in isolation, as gelsolin is cleaved during apoptosis into its two halves by members of the caspase family of proteases. ${ }^{8}$ G1-G3 joins the process of preparing a cell for death by dismantling its actin-based filament systems in a manner that is free from direct regulation by calcium ions. Isolated G1-G3 is capable 
of severing actin filaments in a calcium-independent manner that can be attributed to an initial attachment of G2 followed by a sequence of contacts with actin that pry open the G1-G3 latch. ${ }^{5}$

In spite of the absence of strict regulation of the actin filament-severing ability of G1-G3, calcium ion binding to this fragment does influence its structure and activity, as demonstrated by a reduced hydrodynamic volume, diminished proteolytic susceptibility, and increased tryptophan fluorescence. ${ }^{9,10}$ Isolated G1 experiences a three-orders of magnitude increase in affinity for actin in the presence of calcium ions, and the $\mathrm{Ca}^{2+}$ occupying its type II site is reported to be locked into the G1-actin structure, inaccessible to chelation by EGTA. ${ }^{11}$ Furthermore, transient binding of $\mathrm{Ca}^{2+}$ to $\mathrm{G} 2$ is proposed to facilitate the activation process. ${ }^{5}$

Isolated G4-G6 retains an absolute requirement for calcium ions, in vitro at neutral $\mathrm{pH}$, with respect to binding actin. ${ }^{12}$ Tryptophan fluorescence and equilibrium dialysis experiments have provided evidence for two high-affinity calcium-binding sites in G4-G6 $\left(K_{\mathrm{d}}\right.$ values of $0.2 \mu \mathrm{M}$ and $\left.2 \mu \mathrm{M}\right){ }^{12}$ Given the lack of requirement for calcium for G1-G3 activity, the occupation of these two sites in G4-G6 is considered to be the minimum requirement to activate full-length gelsolin. A third, low-affinity calcium-binding site in G4-G6, characterized by $K_{\mathrm{d}}=100 \mu \mathrm{M}$, has also been identified from changes in susceptibility to chemical or enzymatic modification. ${ }^{13,14}$

Structural studies have demonstrated that there is a conserved calcium-binding site in each of the six gelsolin domains, referred to as type II binding sites. ${ }^{15}$ Hence, biochemical and structural data concur that there are three calcium-binding sites in G4-G6. However, assignment of the calcium-binding sites has been controversial. In isolation, G4-G5 and G5-G6 bind calcium ions with $K_{\mathrm{d}}$ values of $2 \mu \mathrm{M}$ and $0.2 \mu \mathrm{M}$, respectively, while G4 and G5 alone do not bind calcium ions. ${ }^{12}$ This led to assignment of the $2 \mu \mathrm{M}$ site to G4, as this site lies at the interface of G4 with G5.
The $0.2 \mu \mathrm{M}$ site was allocated to G6 due to its favorable location in the structure of inactive gelsolin to spring the tail latch. Finally, the low-affinity site was rationalized to be in G5 where it could stabilize the final activated structure through mediating the G5:G6 interface. ${ }^{15}$ In contrast, a structure of the calcium-bound form of G4-G6 suggested that the two high-affinity calcium-binding sites lie in G5 and G6. ${ }^{16}$ However, a higher-resolution structure of calcium activated G4-G6 revealed three bound calcium ions. ${ }^{17}$ Re-evaluation of the data for the first structure led to the conclusion that all three calcium-binding sites are filled in both structures and neither structure provides strong evidence relating to the affinities for the calcium-binding sites. More recently, protease susceptibility studies have demonstrated protection of the loop between G5 and G6 at above $50 \mu \mathrm{M} \mathrm{Ca}^{2+}$, leading the authors to conclude that the low-affinity calcium-binding site lies in G5. ${ }^{13}$

Here, we probe the calcium-binding sites of the $\mathrm{N}$ and C-terminal halves of gelsolin by varying the free metal ion environment surrounding protein crystals. We verify two viable type I and six viable type II metal ion binding sites in the crystalline fragments. We also demonstrate the calcium ions bound into the parent crystals to be exchangeable, but find them to be held with modified affinities, within the crystal environment, with respect to those determined in solution. On extraction of calcium ions from G4-G6, we discover that replacement of $\mathrm{Ca}^{2+}$ by water may represent a possible intermediate state in the deactivation of gelsolin.

\section{Results}

\section{Structures of G4-G6}

G4-G6 crystals grown at $\mathrm{pH} 4.5$ were washed repeatedly in calcium-free solutions at $\mathrm{pH} 4.5$ or 8.0 and the structures were determined (Figure 1 and
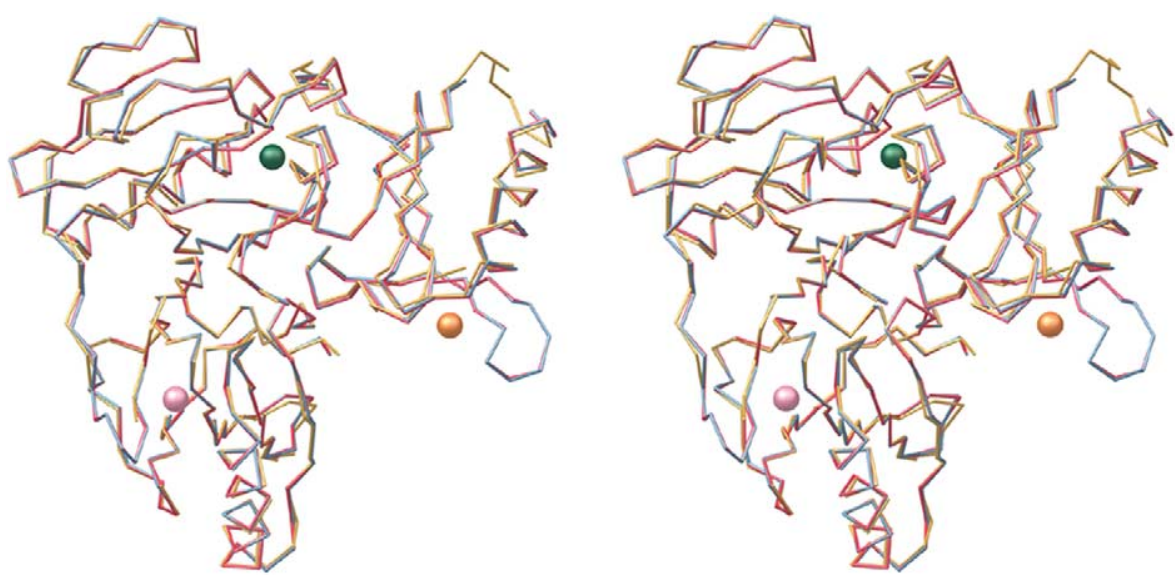

Figure 1. A stereo representation of the polypeptide backbone of G4-G6. Red: from crystals grown at $\mathrm{pH} 4.5$ and subjected to initial washing conditions at $\mathrm{pH} 4.5$. Blue: from crystals grown at $\mathrm{pH} 4.5$ and subjected to initial washing conditions at $\mathrm{pH}$ 8.0. Gold: from crystals grown at $\mathrm{pH} 8.0$ and subjected to EGTA soaking conditions at that $\mathrm{pH}$. Spheres coloured pink, green and orange represent calcium ions in the type II sites of G4, G5 and G6, respectively, in G4-G6 crystals grown at and subjected to initial washing conditions at $\mathrm{pH} 4.5$. 
Table 1. Data collection and refinement statistics for the G4-G6 structures

\begin{tabular}{|c|c|c|c|c|}
\hline & Initial soak pH 4.5 & EGTA soak pH 4.5 & Initial soak pH 8.0 & EGTA soak pH 8.0 \\
\hline Wavelength $(\AA)$ & 0.957 & 0.950 & 1.095 & 0.970 \\
\hline Space group & $P 2_{1} 2_{1} 2_{1}$ & $P 2_{1} 2_{1} 2_{1}$ & $P 2_{1} 2_{1} 2_{1}$ & $P 2_{1} 2_{1} 2_{1}$ \\
\hline \multirow[t]{2}{*}{ Unit cell } & $a=84.8, b=90.3$ & $a=85.3, b=90.9$ & $a=85.1, b=90.5$ & $a=84.1, b=87.8$ \\
\hline & $\begin{array}{c}c=156.3 \AA \\
\alpha=\beta=\gamma=90^{\circ}\end{array}$ & $\begin{array}{c}c=157.6 \AA \\
\alpha=\beta=\gamma=90^{\circ}\end{array}$ & $\begin{array}{c}c=156.6 \AA \\
\alpha=\beta=\gamma=90^{\circ}\end{array}$ & $\begin{array}{c}c=155.7 \AA \\
\alpha=\beta=\gamma=90^{\circ}\end{array}$ \\
\hline Resolution range $(\AA)$ & $20.0-1.55(1.59-1.55)$ & $20.0-2.50(2.56-2.50)$ & $20.0-2.87(2.94-2.87)$ & $\begin{array}{c}\alpha=\beta=\gamma=90 \\
29.4-3.0(3.08-3.00)\end{array}$ \\
\hline Total reflections & $693,903(66,798)$ & $402,916(39,135)$ & $103,525(19,489)$ & $93,285(13,794)$ \\
\hline Unique reflections & $173,539(17,192)$ & $42,890(4235)$ & $27,880(5590)$ & $22,390(1680)$ \\
\hline Redundancy & $4.0(3.9)$ & $9.4(9.3)$ & $3.7(3.5)$ & $4.1(3.9)$ \\
\hline Completeness (\%) & $99.9(99.7)$ & $99.7(99.5)$ & $98.4(85.3)$ & $99.5(99.9)$ \\
\hline Average $I / \sigma$ & $29.2(3.4)$ & $23.3(4.7)$ & $12.5(7.9)$ & $19.4(5.1)$ \\
\hline$R_{\text {merge }}(\%)$ & $4.2(37.6)$ & $8.0(37.4)$ & $4.5(9.0)$ & $6.2(23.0)$ \\
\hline$R_{\text {factor }}(\%)$ & $19.1(24.0)$ & $20.4(28.6)$ & $20.0(30.1)$ & $21.4(30.3)$ \\
\hline$R_{\text {free }}{ }^{\mathrm{c}}(\%)$ & $21.4(27.7)$ & $25.5(33.8)$ & $26.2(35.6)$ & $31.0(44.3)$ \\
\hline $\begin{array}{l}\text { Non-hydrogen atoms } \\
\text { (calcium, water) }\end{array}$ & $8675(9,1170)$ & $7836(8,332)$ & $7719(9,215)$ & $7571(0,67)$ \\
\hline $\begin{array}{l}\text { Molecule A consists of } \\
\text { residues }\end{array}$ & $\begin{array}{c}412-635,638-707 \\
715-741\end{array}$ & $\begin{array}{c}412-635,638-707 \\
715-741\end{array}$ & $\begin{array}{c}412-635,638-707 \\
715-741\end{array}$ & $\begin{array}{l}412-525,530-635,638- \\
645,656-707,715-741\end{array}$ \\
\hline $\begin{array}{l}\text { Molecule B consists of } \\
\text { residues }\end{array}$ & $414-454,459-741$ & $414-454,459-741$ & $414-454,459-741$ & $\begin{array}{c}414-454,459-524 \\
530-741\end{array}$ \\
\hline $\begin{array}{l}\text { Molecule } C \text { consists of } \\
\text { residues }\end{array}$ & $414-453,460-741$ & $414-453,460-741$ & $414-453,460-741$ & $\begin{array}{c}414-453,460-525 \\
530-741\end{array}$ \\
\hline $\begin{array}{l}\text { Mean temperature factor } \\
\text { for each molecule }\left(\AA^{2}\right)\end{array}$ & $22.7,23.5,28.0$ & $37.1,39.0,44.6$ & $27.2,28.2,33.5$ & $66.3,52.2,67.8$ \\
\hline RMS deviation bonds $(\AA)$ & 0.005 & 0.012 & 0.008 & 0.012 \\
\hline RMS deviation angles (deg.) & 1.45 & 1.26 & 1.09 & 1.35 \\
\hline $\begin{array}{l}\text { a } R_{\text {merge }}=(\Sigma|I-\langle I\rangle| / \Sigma\langle I\rangle) . \\
\text { b } R_{\text {factor }}=\left(\Sigma\left|F_{\mathrm{o}}\right|-\left|F_{\mathrm{c}}\right| / \Sigma\left|F_{\mathrm{o}}\right|\right) \\
\mathrm{c} \text { Based on } 5 \% \text { of the data. }\end{array}$ & & & & \\
\hline
\end{tabular}

Table 1). The crystals were of the same space group, having three molecules per asymmetric unit and similar cell dimensions, as those found previously for G4-G6 crystals grown in $1 \mathrm{mM} \mathrm{CaCl}_{2}$ at $\mathrm{pH} 7.5$. ${ }^{17}$ These three structures are essentially identical. All three type II calcium-binding sites in each of the G4G6 molecules in the asymmetric unit display appropriate electron density and coordination to assert unequivocally that they are occupied by metal ions (Tables 2 and 3). These ions are consistent with being $\mathrm{Ca}^{2+}$ due to the crystallization conditions and reasonable $B$-factors on refinement as such (Table 2).
Inclusion of $1.0 \mathrm{mM}$ EGTA in the washing media at $\mathrm{pH} 8.0$ was able to remove calcium ions from type II calcium-binding sites in each of domains G4 (Figure 2), G5 (Figure 3) and G6 (Figure 4) (Table 1). Residual calcium ions could be detected only in one of the three copies of G5 (in molecule B) in the asymmetric unit. The overall structure of the EGTAextracted G4-G6 was that determined in the presence of calcium (Figure 1), however, local conformational changes were observed around the vacated calcium-binding sites. Loss of calcium from the G4 type II site results in the release of the Thr524 carbonyl group from the metal ion-binding site and

Table 2. Characterization of the occupancy of the metal-binding sites

\begin{tabular}{|c|c|c|c|c|c|c|c|}
\hline \multirow[b]{2}{*}{ Domain } & \multicolumn{2}{|c|}{ High calcium pH 7.5 (1P8X) } & \multicolumn{2}{|c|}{ Initial soak pH 4.5} & \multicolumn{2}{|c|}{ EGTA pH 4.5} & \multirow{2}{*}{$\begin{array}{c}\text { Terbium } \\
\text { pH } 4.5 \\
\begin{array}{c}\text { Peak height } \\
(\sigma)\end{array}\end{array}$} \\
\hline & $B$-factor $\left(\AA^{2}\right)$ & $\begin{array}{l}\text { Peak height } \\
(\sigma)\end{array}$ & $B$-factor $\left(\AA^{2}\right)$ & $\begin{array}{l}\text { Peak height } \\
(\sigma)\end{array}$ & $B$-factor $\left(\AA^{2}\right)$ & $\begin{array}{l}\text { Peak height } \\
(\sigma)\end{array}$ & \\
\hline G4-A & 24.0 & 14.2 & 19.3 & 28.2 & 27.2 & 14.2 & 21.2 \\
\hline G4-B & 22.6 & 15.1 & 18.7 & 25.3 & 27.5 & 14.8 & 29.6 \\
\hline G4-C & 40.4 & 12.7 & 25.3 & 18.0 & 31.8 & 12.1 & 18.2 \\
\hline G5-A & 19.5 & 15.8 & 17.8 & 31.9 & 26.8 & 14.9 & 27.0 \\
\hline G5-B & 21.6 & 15.5 & 17.7 & 31.4 & 25.3 & 14.5 & 28.0 \\
\hline G5-C & 25.7 & 14.4 & 21.5 & 23.2 & 30.5 & 11.7 & 24.8 \\
\hline G6-A & 29.4 & 13.2 & 17.1 & 31.1 & 26.9 & 14.8 & 16.5 \\
\hline G6-B & 28.8 & 13.7 & 22.0 & 23.7 & 35.1 & 9.0 & 23.1 \\
\hline G6-C & 35.6 & 9.3 & 25.9 & 14.6 & - & 2.7 & 21.7 \\
\hline G4-Avg & 29.0 & 13.8 & 21.1 & 23.8 & 28.9 & 13.7 & 23.0 \\
\hline G5-Avg & 22.3 & 15.2 & 19.0 & 28.8 & 27.5 & 13.7 & 26.6 \\
\hline G6-Avg & 31.3 & 12.1 & 21.7 & 23.1 & $31.0^{\mathrm{a}}$ & 8.8 & 20.4 \\
\hline
\end{tabular}


Table 3. Characterization of the geometries of the metal-binding sites

\begin{tabular}{|c|c|c|c|c|c|c|}
\hline \multirow[b]{2}{*}{ Domain } & \multirow[b]{2}{*}{ Residue } & \multirow[b]{2}{*}{ Atom } & \multicolumn{4}{|c|}{ Average distance to calcium ion $(\AA)$} \\
\hline & & & $\begin{array}{l}\text { High calcium } \\
\text { pH } 7.5(1 \mathrm{P} 8 \mathrm{X})\end{array}$ & $\begin{array}{l}\text { Initial soak } \\
\mathrm{pH} 4.5\end{array}$ & & pH 4.5 \\
\hline \multirow[t]{5}{*}{ G4 } & Gly444 & $\mathrm{O}$ & 2.43 & 2.33 & 2.39 & \\
\hline & Asp445 & OD1 & 2.35 & 2.34 & 2.36 & \\
\hline & Glu475 & OE1 & 2.53 & 2.40 & 2.40 & \\
\hline & Glu475 & OE2 & 2.69 & 2.66 & 2.52 & \\
\hline & Thr524 & $\mathrm{O}$ & 2.53 & 2.38 & 2.39 & \\
\hline \multirow[t]{5}{*}{ G5 } & Asn564 & $\mathrm{O}$ & 2.46 & 2.34 & 2.28 & \\
\hline & Asn564 & OD1 & 2.47 & 2.38 & 2.32 & \\
\hline & Asp565 & OD1 & 2.45 & 2.35 & 2.33 & \\
\hline & Glu587 & OE1 & 2.74 & 2.62 & 2.70 & \\
\hline & Glu587 & OE2 & 2.50 & 2.35 & 2.39 & \\
\hline \multirow[t]{4}{*}{ G6 } & Asp669 & $\mathrm{O}$ & 2.28 & 2.31 & $2.26^{\mathrm{a}}$ & $2.24^{\mathrm{b}}$ \\
\hline & Asp670 & OD2 & 2.60 & 2.40 & $2.64^{\mathrm{a}}$ & $3.94^{\mathrm{b}}$ \\
\hline & Glu692 & OE1 & 2.63 & 2.57 & $2.47^{\mathrm{a}}$ & $2.51^{\mathrm{b}}$ \\
\hline & Glu692 & OE2 & 2.44 & 2.45 & $2.43^{\mathrm{a}}$ & $2.63^{\mathrm{b}}$ \\
\hline
\end{tabular}

Mean bond distances are given for the ligating atoms within each domain, which are given as an average of the three molecules in the asymmetric unit. The boxed value highlights a non-bonding distance.

a The average for only molecules A and B.

b Values for molecule C alone.

the disordering of the proximal loop (residues 526528 , Figure 2(b)). Removal of calcium from the G5 type II site showed little change in the coordinating residues or the relative orientations of G5 as compared to G6 (Figure 3). Molecule B showed some retention of calcium at this site judged by a peak of $4.0 \sigma$ in the $2 F_{\mathrm{o}}-F_{\mathrm{c}}$ electron density map. Finally, removal of calcium from the type II site in G6 showed a reduced peak in the $2 F_{\mathrm{o}}-F_{\mathrm{c}}$ electron density map close to the G6 type II calcium-binding site (Figure 5(b)). Amino acid side-chain and water molecule positions around this site are inappropriate to support calcium ion coordination. Instead, the coordination and electron density are consistent with a water molecule forming a hydrogen bonded bridge between the carboxylate of Glu692 and the carbonyl oxygen of Asp669, with the carboxylate of Asp670 withdrawn by approximately $1.5 \mathrm{~A}$ from the calcium ion-binding site (Figure 5). Washing crystals with EGTA-containing solutions at $\mathrm{pH} 4.5$, consistent with protonation of EGTA and a reduced affinity for $\mathrm{Ca}^{2+}$, resulted in the extraction of none of the calcium ions with the exception of the type II site of G6 from molecule C (Figure 5(b), Tables 2 and 3). This $\mathrm{pH} 4.5 \mathrm{G} 6$ calcium-free conformation showed the same features as that determined at $\mathrm{pH} 8.0$ in the presence of EGTA.

In an attempt to exchange rather than extract calcium ions from G4-G6, the crystals were washed in solutions lacking $\mathrm{Ca}^{2+}$ and EGTA, but containing (a)

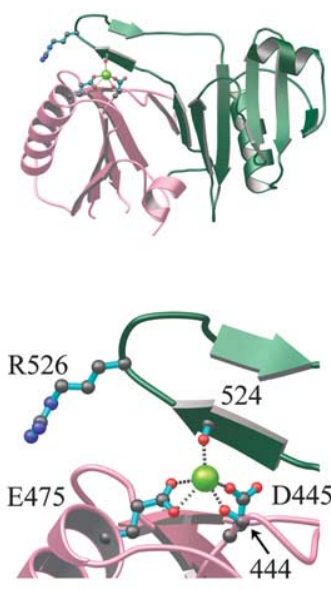

Low Calcium (b)

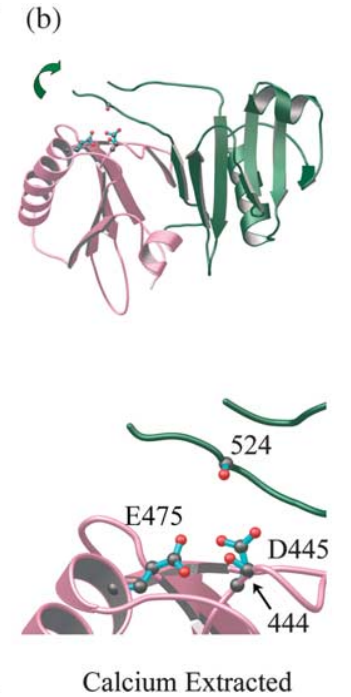

(c)

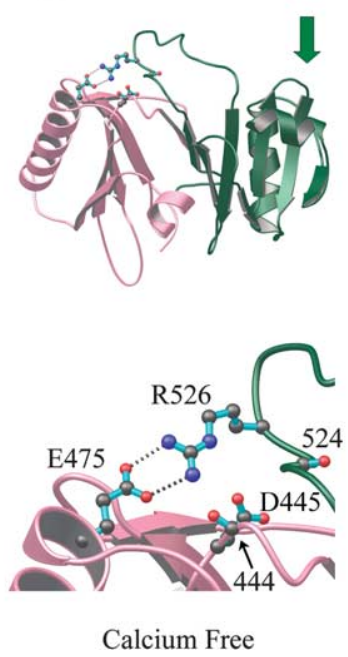

Figure 2. Structural consequences of type II calcium binding by G4. (a) G4-G5, with the type II calciumbinding site of G4 enlarged in the lower part of the panel, taken from the high-resolution structure of G4G6 subjected to the initial wash conditions at $\mathrm{pH} 4.5$ (low calcium). G4 is depicted in pink and G5 in dark green. The G4 type II calcium ion is shown as a light green sphere and the ligating residues are drawn in ball-and-stick format. Arg526 also is depicted. (b) G4-G5, with the type II site of G4 enlarged in the lower part of the panel, taken from the structure of G4-G6 subjected to the EGTA soaking conditions at pH 8.0 (calcium extracted). Also depicted are the residues from (a). (c) G4-G5, with the type II site of G4 enlarged in the lower part of the panel, excised from the calcium-free structure of gelsolin (PDB i.d. 1D0N) ${ }^{6}$ is depicted with the residues from (a). Here, Arg526 forms an ion-pair interaction with Glu475, which is a calcium ion ligand in (a). The green arrows highlight changes in the folding of the backbone of G5 on moving from (a) through to (c). 
(a)

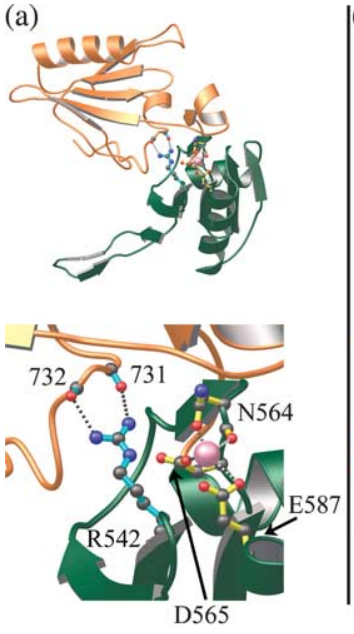

Low Calcium

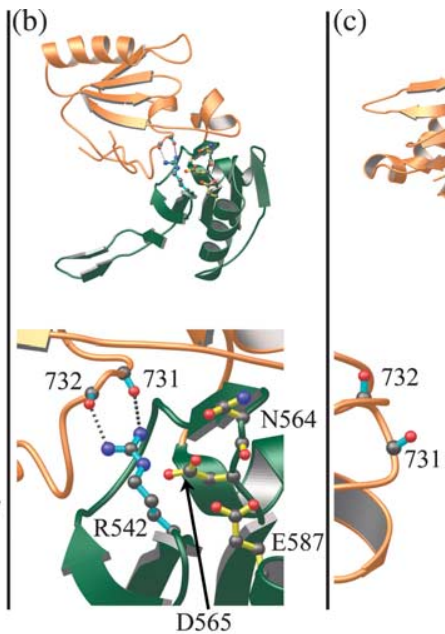

Calcium Extracted

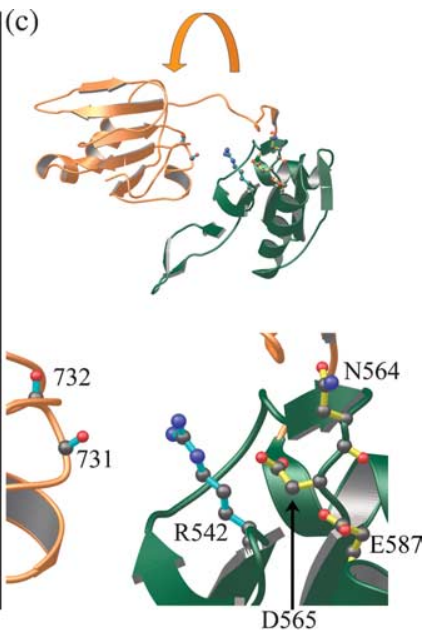

Calcium Free

Figure 3. Structural consequences of type II calcium binding by G5. (a) G5-G6, with the type II calciumbinding site of G5 enlarged in the lower part of the panel, taken from the high-resolution structure of G4G6 subjected to initial washing conditions at pH 4.5 (low calcium). The calcium ion (pink) coordinating residues are drawn. Broken lines indicate electrostatic interactions of the side-chain of Arg542 with mainchain carbonyl groups at Gly731 and Trp732. ${ }^{6}$ (b) G5-G6, with the type II site of G5 enlarged in the lower part of the panel, taken from the structure of G4-G6 subjected to EGTA soaking conditions at $\mathrm{pH} 8.0$ (calcium extracted). Colours and residues are as in (a). (c) G5-G6, with the type II site of G5 enlarged in the lower part of the panel, from the calciumfree form of gelsolin (PDB i.d. $1 \mathrm{D} 0 \mathrm{~N}){ }^{6}$ is shown in the same manner as (a). The orange curved arrow indicates the rotation of G6 relative to G5 that would be necessary to reach the inactive form of gelsolin from the activated form in (a).

$1.0 \mathrm{mM} \mathrm{Tb}{ }^{3+}$. Anomalous difference electron density maps were calculated after minimal refinement of the G4-G6 structure (Figure 6(a) and Table 1). Peaks greater than $15 \sigma$ were observed at each of the type II calcium-binding sites (Table 4) at both $\mathrm{pH} 4.5$ and $\mathrm{pH}$ 8.0. Backwashing crystals that had been subjected to terbium soaking conditions with solutions containing excess $\mathrm{Ca}^{2+}$ resulted in loss of signal due to $\mathrm{Tb}^{3+}$ in all cases, confirming the exchangeability of the ions bound in the G4-G6 crystals.

Furthermore, low, but significant, occupancies were observed of the G4 type I calcium-binding site (18.17 $\sigma$ and $6.31 \sigma$, respectively, in molecules $A$ and $\mathrm{B}$ at $\mathrm{pH}$ 4.5). Type I calcium-binding sites previously have been reported to be occupied only in structures of complexes containing both gelsolin and actin, sandwiched between G1 or G4 and actin. ${ }^{15,18}$ No other peaks were seen above $5 \sigma$ in these maps (Figure 6(a)). The identification of these type I sites as genuine metal ion-binding sites, even when lacking the negative charge of Glu167 from actin, encouraged re-examination of calciumbound structures already discussed here. Peak heights of $6.09 \sigma$ and $4.39 \sigma$ exist for the G4 type I site in the $2 F_{\mathrm{o}}-F_{\mathrm{c}}$ electron density map for molecules $\mathrm{B}$ and $\mathrm{C}$, respectively, in crystals subjected to the initial wash conditions at $\mathrm{pH} 4.5$. These values are significant, but lower than those observed for the type II sites in the same crystal (Table 2), suggesting a proportionately lower, but not negligible level of calcium occupancy. Similar (a)

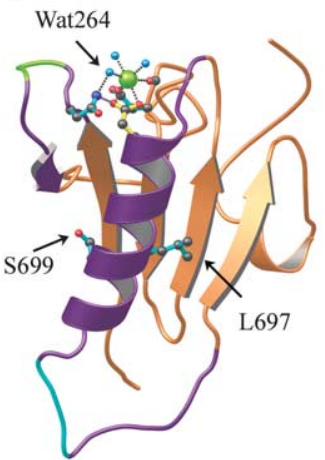

Low Calcium (b)

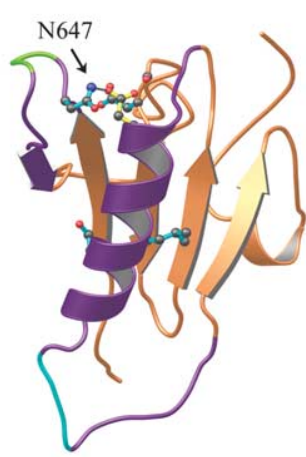

Calcium Extracted (c)

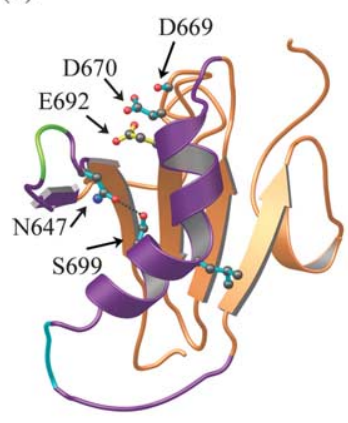

Calcium Free
Figure 4. Structural consequences of type II calcium binding by G6. (a) G6 taken from the high-resolution structure of G4-G6 subjected to initial washing conditions at $\mathrm{pH}$ 4.5. The calcium ion (green) coordinating residues and water molecules (blue) are drawn. Broken lines indicate the coordination. Residue Asn647 forms a hydrogen bond to water 264 (Wat264), which itself is part of the coordination sphere. An enlarged image of the G6 type II calcium-binding site, with important residues labeled, is found in Figure 5(a). Structure coloured purple represents regions of G6 that undergo conformational change on binding calcium. Chain coloured green or cyan is involved in contacts with G1-G3 in the calcium-free form of gelsolin (PDB i.d. 1DON). ${ }^{6}$ Chain coloured green is additionally involved in actin interactions in G4-G6/actin (PDB i.d. 1H1V). ${ }^{15}$ Two residues on the G6 main helix also are shown, Leu697 and Ser699. (b) G6 taken from the structure of G4-G6 subjected to EGTA soaking conditions at $\mathrm{pH}$ 8.0. Colours and residues are as in (a). An enlarged image of the G6 type II site in this structure, with important residues labeled, is found in Figure 5(b). (c) G6 from the calcium-free form of gelsolin is shown in the same manner as in (a). Notably, Asn647 forms a hydrogen bond with Ser699 in this structure. 


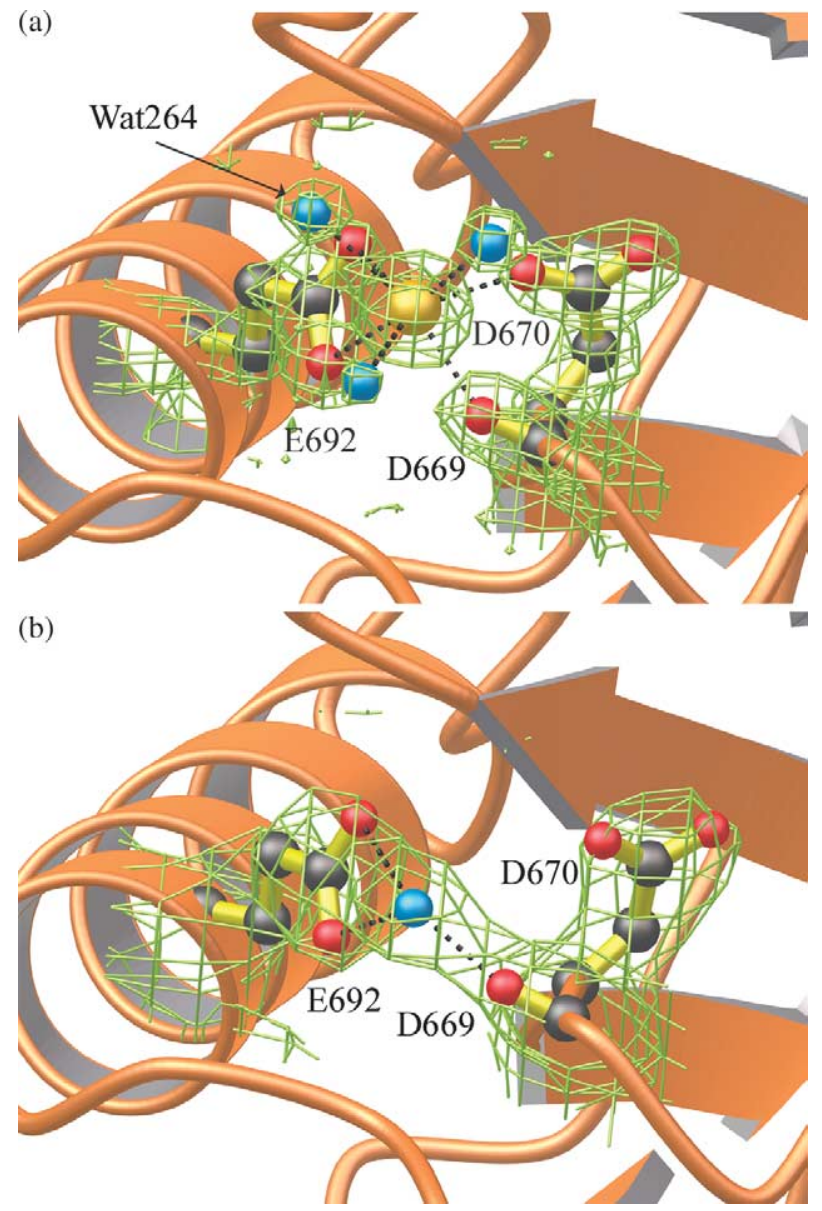

Figure 5. Removal of calcium from a G6 type II site. (a) A close-up of the type II calcium-binding site in G6 taken from the high-resolution structure of G4-G6 subjected to initial wash conditions at $\mathrm{pH} 4.5$. The $2 F_{\mathrm{o}}-$ $F_{\mathrm{c}}$ electron density map (green), contoured at $2 \sigma$, is displayed around the main features within the calciumbinding region. (b) A close-up of the same region from the crystals subjected to EGTA wash conditions at $\mathrm{pH}$ 4.5. The $2 F_{\mathrm{o}}-F_{\mathrm{c}}$ electron density map, contoured at $1 \sigma$, shows that Asp670 has moved away from the calcium-binding site and that a water molecule, Wat264, is coordinated instead by Glu692 and the main-chain carbonyl group of Asp670. Note that to most clearly depict coordination of $\mathrm{Ca}^{2+}$ in (a) and of water in (b), the orientations have been altered from those presented in Figure 4(a) and (b).

results were obtained at $\mathrm{pH}$ 8.0, however, all EGTA soaks showed zero occupancy of the G4 type I calcium-binding site.

\section{Calcium exchange in G1-G3/actin}

Two crystal forms of ordered G1-G3/actin were grown from solutions of the complex of full-length gelsolin bound to two actin molecules (Table 4). The $P 3_{1} 21$ form had been shown to contain G1-G3 bound to a single actin molecule resulting from proteolytic cleavage during crystallization. ${ }^{5}$ The second crystal form, $P 6_{5} 22$, in spite of a larger unit cell volume and the absence of detectable proteolysis, showed a molecular replacement solution only for G1-G3/actin. The packing of G1-G3/actin is sufficient to construct the entire crystal with space between the ordered G1-G3/actin regions to accommodate multiple orientations of G4-G6/ actin (unpublished results).

Washing both of these crystal forms containing G1-G3/actin with $\mathrm{Tb}^{3+}$ solutions yielded anomalous difference electron density maps in which all three type II metal ion-binding sites are occupied by terbium ions (Figure 7(a)). A terbium ion also replaces the calcium ion previously detected in the type I site in both types of crystal. No other significant peaks were observed in the $\mathrm{P}_{5} 22$ anomalous difference electron density map, confirming the correctness of the molecular replacement solution. Interestingly, while $\mathrm{Tb}^{3+}$ clearly replaced the ATP-associated $\mathrm{Ca}^{2+}$ in the $P 3_{1} 21$ crystal soaked at $1 \mathrm{mM} \mathrm{Tb}{ }^{3+}$ it failed to do so in the second crystal form soaked at $0.2 \mathrm{mM} \mathrm{Tb}^{3+}$. Incubation of the $\mathrm{P}_{5} 22$ crystals in increasing ratios of calcium:terbium ions effectively competed away the $\mathrm{Tb}^{3+}$ from the type II sites in G1 and G3. However, even at a 50:1 ratio $\left(10 \mathrm{mM} \mathrm{Ca}^{2+}: 0.2 \mathrm{mM}\right.$ $\mathrm{Tb}^{3+}$ ) the G1 type I and the G2 type II site reproducibly showed strong anomalous electron density due to $\mathrm{Tb}^{3+}$ (Figure $7(\mathrm{~b})$ ).

\section{Discussion}

\section{Overall structure of G4-G6}

Here, we have demonstrated that the structure of calcium-bound G4-G6 determined from crystals grown at $\mathrm{pH} 4.5$ is essentially identical with that determined at $\mathrm{pH} 7.5$. $^{17}$ This finding indicates that the combination of activators, low $\mathrm{pH}$ and calcium, do not induce conformational changes in the C-terminal half of the molecule beyond those provoked by calcium alone. Furthermore, extraction of calcium ions from crystalline G4-G6 did not return the molecule to its calcium-free conformation (Figures 2-4) but locked G4-G6 in the activated conformation via the crystal contacts. This stabilization of G4-G6 in an activated yet calcium-free form provides an opportunity to assess the local conformational changes around the vacated calcium-binding sites, changes that may represent snapshots of the early events in the deactivation of gelsolin.

\section{Type II sites in G4-G6}

The three calcium ions in the type II sites in the C-terminal half of gelsolin, one in each of G4, G5 and G6, were observed to readily exchange with terbium ions and were able to be extracted with EGTA at $\mathrm{pH} 8.0$ in line with solution studies. ${ }^{12}$ Removal of calcium from the type II site in G4 resulted in the extraction of residue Thr524 from the metal ion-binding site and the disordering of 


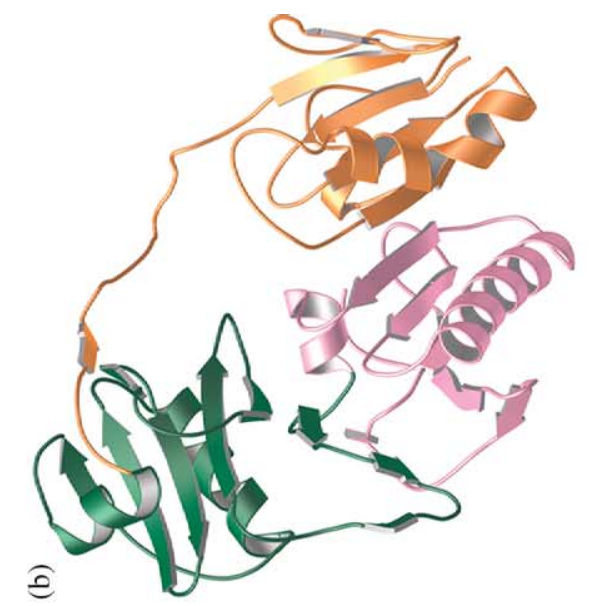

of the adjacent loop (residues 526-528; Figure 2(a) and
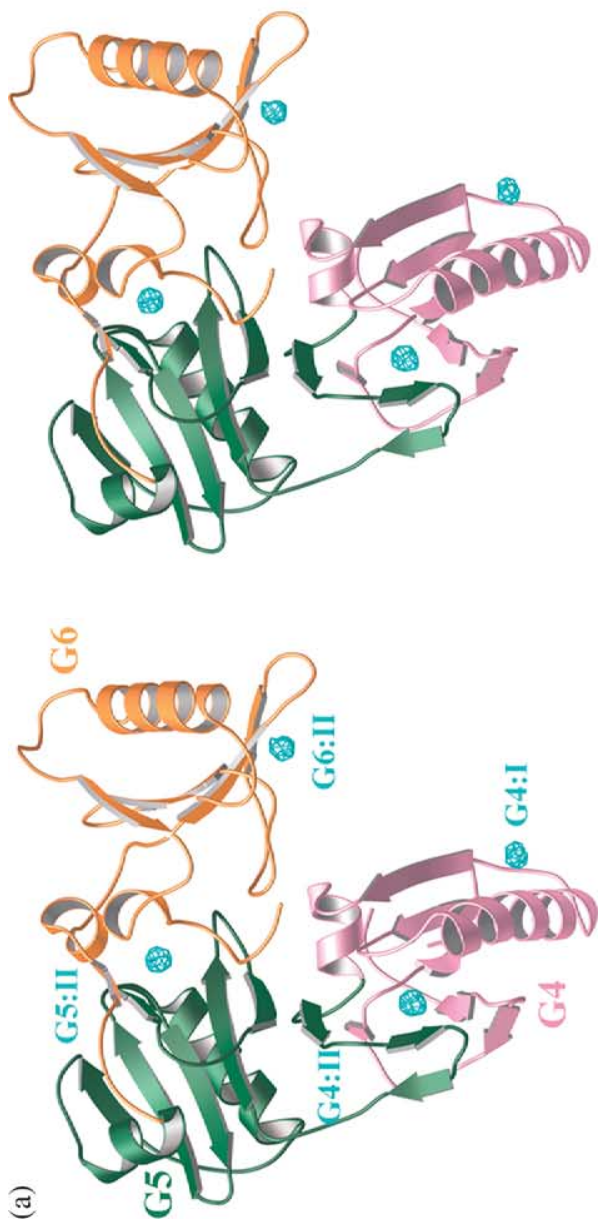

the adjacent loop (residues 526-528; Figure 2(a) and
(b)). This loop contains Arg526, which in the inactive molecule interacts with Glu475 (Figure 2(c)). Glu475 forms part of the calcium coordination in the activated molecule (Figure 2(a)). Hence, loss of calcium at this site leads to loop flexibility allowing Arg526 to partially replace the calcium interactions culminating in a shift in the relative orientation of G4 with respect to G5 (Figure 2). The adjustment to the position of G5 relative to G4 translates the start of the G5-G6 linker by $10 \AA$. This movement will provide tension in the G5-G6 linker and destabilize the common G4:G6 $\beta$-sheet (Figure 6(b)).

In contrast, removal of calcium from the type II site in G5 resulted in only minor changes in a few side-chain residues (Figure $3(\mathrm{a})$ and (b)). This calcium ion lies at the centre of the interface between G5 and G6, an interface that is absent from the inactive molecule (Figure 3(c)). Crystal contacts lock the G5-G6 interaction, preventing the dissociation of G6 from G5 that would result from the loss of calcium stabilization of this interface. Indeed, one molecule of G4-G6 within the crystal asymmetric unit at least partially retained its G5bound calcium in the presence of EGTA. Furthermore, the initial soak conditions, in which calcium was observed in this site, contained calcium levels far below that reported for occupation of this site. ${ }^{13}$ Thus, stabilization of the G5-G6 interface appears to increase the affinity of the G5 type II site for calcium. The interaction of G4-G6 with actin may also reasonably be expected to stabilize the G5-G6 interface leading to a modulation of the affinity of this site in the presence of actin. ${ }^{15}$

Loss of calcium from the type II binding site in G6 led to the ordering of a water molecule by two of the calcium coordination sphere residues, Glu692 and Asp669, while Asp670 moved away from the site (Figures 4(a) and (b) and 5). This observation provides an intriguing insight into the mechanism of deactivation of gelsolin, whereby the replacement of the calcium by water and the release of part of the coordination sphere may be a prerequisite for larger scale conformational changes throughout gelsolin. Curiously, at pH 4.5, EGTA was able to extract a calcium ion from the G6 type II site in only one molecule of the crystal asymmetric unit (molecule C), while all other type II sites remained occupied. EGTA is a poor chelator of calcium at this $\mathrm{pH}$, and as such, these are mild extraction conditions, suggesting that the G6 site in molecule $\mathrm{C}$ has a lower affinity for calcium than all the other sites. The long helix of G6 (purple in Figure 4(a)) in molecule $C$ is slightly shifted with respect to the central $\beta$-sheet in comparison to the two other molecules in the crystal asymmetric unit (not shown). This helix provides part of the calcium coordination sphere, Glu692 (Figure 5). Hence, holding the helix in a sub-optimal conformation lowers the affinity for calcium. In the absence of crystal contacts, we propose that the opposite mechanism prevails. Binding of calcium to the 
Table 4. Data collection and molecular replacement statistics for terbium-soaked crystals

\begin{tabular}{|c|c|c|c|}
\hline & G1-G3/actin & G1-G3/actin & G4-G6 \\
\hline Wavelength $(\AA)$ & 0.970 & 0.974 & 0.951 \\
\hline Space group & $P 3_{1} 21$ & $P 6_{5} 22$ & $P 2_{1} 2_{1} 2_{1}$ \\
\hline Unit cell $(a, b, c: \AA)$ & $a=b=144.6, c=128.3$ & $a=b=146.3, c=388.8$ & $a=85.0, b=90.5, c=155.7$ \\
\hline$(\alpha, \beta, \gamma:$ deg. $)$ & $\alpha=\beta=90, \gamma=120$ & $\alpha=\beta=90, \gamma=120$ & $\alpha=\beta=\gamma=90$ \\
\hline Resolution range $(\AA)$ & $30.0-3.50(3.69-3.50)$ & $30.0-6.80(7.17-6.80)$ & $30.0-2.50(2.60-2.50)$ \\
\hline Total reflections & $54,943(4035)$ & $41,945(6261)$ & $203,945(23,756)$ \\
\hline Unique reflections & $17,781(1659)$ & $4492(659)$ & $41,367(4845)$ \\
\hline Redundancy & $3.1(2.4)$ & $9.3(9.5)$ & $3.5(2.7)$ \\
\hline Completeness (\%) & $88.9(58.1)$ & $96.0(100.0)$ & $97.8(90.2)$ \\
\hline Average $I / \sigma$ & $15.7(3.3)$ & $14.7(5.2)$ & $6.0(3.7)$ \\
\hline$R_{\text {merge }}(\%)$ & $6.2(26.3)$ & $13.2(40.3)$ & $7.1(9.6)$ \\
\hline$R_{\text {factor }}(\%)$ & 32.4 & 42.0 & 38.9 \\
\hline Correlation coefficient $^{\mathrm{b}}(\%)$ & 72.9 & 61.8 & 63.3 \\
\hline
\end{tabular}

type II site in inactive G6 (Figure 4(c)) induces straightening of the long helix (purple in Figure 4), which in turn, alters the position of G4 interacting residues (such as Leu697) leading to the destabilization of the G4:G6 common $\beta$-sheet and the activation of G4-G6 (Figure 6).

\section{Type II sites in G1-G3}

The calcium ion type II sites in the N-terminal half of gelsolin, within the G1-G3/actin complex, were also shown to be able to be occupied by terbium ions (Figure 7(a)). Terbium replaces calcium in the type II sites of G1 and G3. However, the type II site of G2 does not bind calcium in the G1-G3/actin conformation, and hence, terbium fills a vacant site in this domain. ${ }^{5}$ Furthermore, consistent with the lack of calcium occupancy of this site, in the G1-G3/actin structure, backwashing the $\mathrm{Tb}^{3+}$-containing crystals with a $10 \mathrm{mM}$ $\mathrm{Ca}^{2+}$ solution fails to remove this particular terbium ion from the structure (Figure 7(b)). These observations confirm the existence of a valid type II metal ion-binding site in G2, which does not bind calcium in the G1-G3/actin conformation. ${ }^{15,19}$ This result is consistent with the mechanism whereby transient binding of calcium at the G2 site destabilizes inactive gelsolin and subsequently the ion is released by the stabilized active structure. ${ }^{5}$

The type II ("intramolecular") $\mathrm{Ca}^{2+}$-binding site in the G1 has been proposed to contain a trapped calcium ion in the presence of actin. ${ }^{11}$ In the G1-G3/ actin complex this particular calcium ion is readily exchanged for a terbium ion (Figure 7). Furthermore, (a)

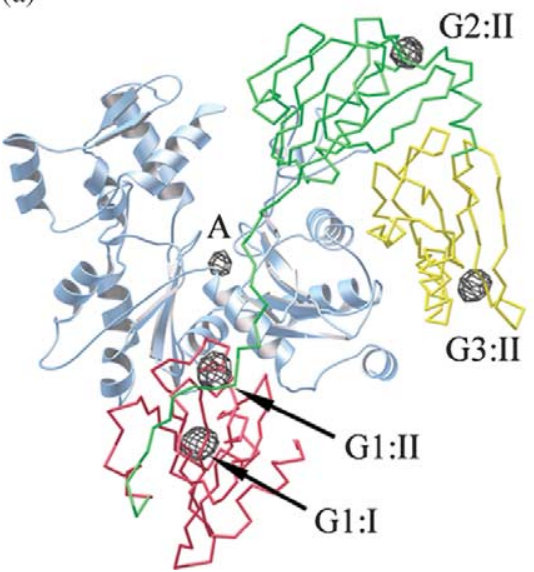

(b)

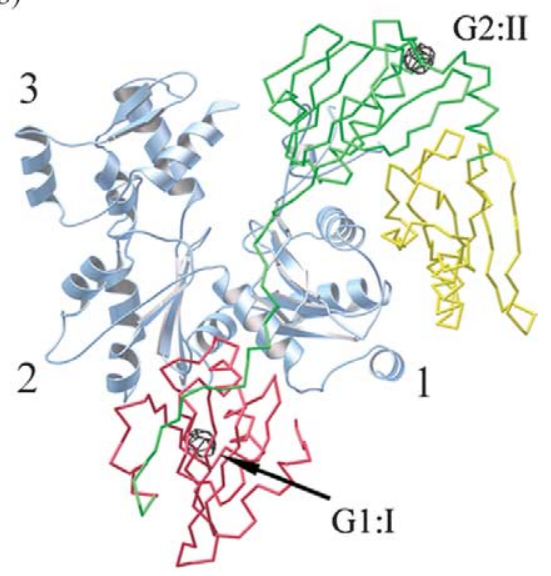

Figure 7. Terbium ion substitution in G1-G3. (a) A cartoon representation of the structure of G1-G3/actin (PDB i.d. 1RGI). Actin is coloured blue and the domains of gelsolin are coloured: G1, red; G2, light green; and G3, yellow. The $P 3_{1} 21$ terbium anomalous difference electron density map, contoured at $5 \sigma$ (black) and derived from terbium-soaked crystals of G1-G3/actin, is superimposed onto the structure. Three terbium ion positions, characterized by the positive density in this map, are identical with the positions of type II metal ion-binding sites in the G1-G3/actin structure and are labeled G1:II, G2:II and G3:II. A fourth terbium ion (G1:I) is in a position where a type I calcium ion is found in the structure. ${ }^{5}$ An actin-ATP-associated $\mathrm{Tb}^{3+}$ (labelled A) also is indicated. (b) A cartoon representation of the structure of G1-G3/actin, with a $P 6_{5} 22 \mathrm{~Tb}^{3+}$ anomalous electron density map superimposed, as in (a). In this case, the terbium ionsoaked crystals were backwashed with mother liquor containing $10 \mathrm{mM} \mathrm{CaCl} 2$ and $0.2 \mathrm{mM} \mathrm{Tb}\left(\mathrm{NO}_{3}\right)_{3}$. $\mathrm{Tb}^{3+}$ was replaced only at the type II sites in G1 and G3. The type II site in G2, which was unoccupied in the structure of G1-G3/ actin, and the type I site sandwiched between actin and G1 remained occupied by terbium ions. 
backwashing the crystal with $10 \mathrm{mM} \mathrm{Ca}^{2+}$ successfully dislodged the terbium ion from this site. Hence, in the context of G1-G3/actin, the G1 type II calciumbinding site operates in a standard equilibrium fashion.

\section{Type I sites in G1 and G4}

The calcium ion in the type I metal ion-binding site in complexes of G1-G3 with actin can be exchanged for a terbium ion (Figure 5). Interestingly, when terbium-soaked crystals are backwashed with $10 \mathrm{mM}$ $\mathrm{Ca}^{2+}$, the $\mathrm{Tb}^{3+}$ sandwiched between actin and G1 at this site resists replacement (Figure 7(b)). This may simply be another case where the increased charge on the terbium ion renders it the thermodynamically favored ion, consistent with the observation that calcium-binding sites often display a stronger affinity for $\mathrm{Tb}^{3+}$ than for $\mathrm{Ca}^{2+} .20$

Previously, type I calcium ions had only been observed bound at the interface between G1 or G4 and actin. ${ }^{15,18}$ The terbium ion soaks of crystals of G4G6 demonstrate that the type I site in G4 can be at least partially occupied in the absence of actin. In light of this result, inspection of $2 F_{\mathrm{o}}-F_{\mathrm{c}}$ electron density maps determined from terbium-free G4-G6 crystals indicates partial occupancy of the G4 type I site by calcium, at $0-25 \%$, relative to the type II sites. Partial occupancy of the type I sites suggests that calcium ions bound here contribute to the destabilization of the common $\beta$-sheet between G4 and G6 during calcium activation (Figure 6(a)), as previously speculated. ${ }^{6}$ Furthermore, as type I calcium ions are coordinated by residues both from gelsolin and actin in the complex, calcium ion concentration will directly affect the affinity of gelsolin binding to actin.

\section{Materials and Methods}

\section{G4-G6 crystals}

G4-G6 was expressed and purified as described. ${ }^{17}$ A slight modification of the published crystallization conditions resulted in reproducible crystals grown at $\mathrm{pH} 4.5$ rather than at $\mathrm{pH}$ 7.5. Briefly, a solution of G4-G6 at $20 \mathrm{mg} / \mathrm{ml}$ in $150 \mathrm{mM} \mathrm{NaCl}, 1 \mathrm{mM} \mathrm{CaCl}, 10 \mathrm{mM}$ Tris$\mathrm{HCl}(\mathrm{pH} 8.0)$ was mixed 1:1 (v/v) with the precipitant, $20 \%(\mathrm{w} / \mathrm{v})$ PEG 8000, $100 \mathrm{mM}$ sodium acetate ( $\mathrm{pH} 4.5)$ at $4{ }^{\circ} \mathrm{C}$, and subjected to batch crystallization. Crystals appeared after two days and were allowed to grow for two weeks before being transferred into the solutions described below.

Initial soak: A crystal (approximately $0.4 \mathrm{~mm} \times$ $0.2 \mathrm{~mm} \times 0.2 \mathrm{~mm}$ ) was transferred, using a loop that matched its size, into a $5 \mu \mathrm{l}$ drop containing $20 \%(\mathrm{w} / \mathrm{v})$ PEG 8000, 20\% (v/v) glycerol and buffered to $\mathrm{pH} 4.5$ using $100 \mathrm{mM}$ sodium acetate, or to $\mathrm{pH} 8.0$ using $100 \mathrm{mM}$ Tris- $\mathrm{HCl}$. After gently stirring the drop for $30 \mathrm{~s}$, the crystal was transferred to a second drop of the same composition, also devoid of added calcium, and the procedure repeated twice more. The crystal was allowed to incubate for $1 \mathrm{~h}$ prior to transfer to a final drop of the same composition, where it rested for $20 \mathrm{~h}$ before being flash-frozen in liquid $\mathrm{N}_{2}$.
EGTA and terbium soaks: By the protocol described above, crystals were transferred into initial soaking solutions supplemented with either $1 \mathrm{mM}$ EGTA or $1 \mathrm{mM} \mathrm{Tb}\left(\mathrm{NO}_{3}\right)_{3}$. The crystals were flash-frozen after a final $20 \mathrm{~h}$ of incubation.

\section{G1-G3/actin crystals and soaks}

A complex containing a 1:2 gelsolin to actin mole ratio was prepared and subjected to crystallization conditions as reported. ${ }^{5}$ Two different crystal forms were obtained. One, which crystallized in space group $P 3_{1} 21$, was found by electrophoresis to contain G1-G3 and actin in an equimolar complex and yielded the G1-G3/actin structure with PDB i.d. 1RGI. ${ }^{5}$ The second form crystallized in space group $\mathrm{P}_{5} 22$ and electrophoretic analysis of the crystals failed to reveal the existence of protoeolytic fragments of gelsolin or actin (unpublished results).

Crystals of both forms were harvested in loops of appropriate size, transferred to solutions of mother liquor supplemented with glycerol $(20 \%)$ to which $\mathrm{Tb}\left(\mathrm{NO}_{3}\right)_{3}$ had been added to a final concentration of $0.2 \mathrm{mM}\left(\mathrm{P}_{5}{ }_{2} 2\right)$ or $1 \mathrm{mM}\left(P 3_{1} 21\right)$, and soaked for $24 \mathrm{~h}$. Subsequently, the $P 6_{5} 22$ crystals were backsoaked for $12 \mathrm{~h}$ with solutions containing step-wise increases $(0 \mathrm{mM}, 0.1 \mathrm{mM}, 1.0 \mathrm{mM}$ and $10 \mathrm{mM}$ ) of $\mathrm{Ca}^{2+}$ in combination with $0.2 \mathrm{mM} \mathrm{Tb}^{3+}$. Subsequently, all crystals were flash-frozen in their respective soaking conditions.

\section{Data collection and analysis}

X-ray diffraction data were collected at $100 \mathrm{~K}$ at ID 7-11, MAX-Lab synchrotron facility, Lund, Sweden. The data sets were indexed using MOSFLM and scaled and merged in SCALA (Tables 1 and 4). ${ }^{21}$ The pH 7.5 G4-G6 structure (PDB i.d. 1P8X), from which the calcium ions had been removed, was refined as a rigid body against each G4-G6 data set. The resulting models were subject to positional and individual $B$-factor refinement using REFMAC5. ${ }^{21}$ Next, an anomalous difference electron density map was calculated for the G4-G6 terbiumsoaked data set and this structure was not subjected to further refinement.

Similarly, the G1-G3/actin structure (PDB i.d. 1RGI), from which the calcium ions were removed, was refined as a rigid body against the terbium-soaked P3 21 G1-G3/ actin data set before the anomalous difference electron density map was calculated. Finally, the terbium-soaked $\mathrm{P}_{5} 22$ G1-G3/actin data sets were subject to molecular replacement using the G1-G3/actin structure (PDB i.d. 1RGI with calcium ions removed) as a search model in the CCP4 version of AMoRe. ${ }^{21}$ Following rigid body refinement, anomalous difference electron density maps were calculated.

The initial soak and EGTA soaked G4-G6 data sets were put through several rounds of model building and refinement with reference to both standard electron density maps and those enhanced by WARP. ${ }^{21}$ Calcium ions were added prior to the last round of refinement. Model building was carried out in the program $\mathrm{O},{ }^{22}$ and model validation using PROCHECK. ${ }^{21}$ MOLSCRIPT was used in preparing the final Figures. ${ }^{23,24}$

\section{PDB accession numbers}

Atomic coordinates discussed in this work for gelsolin domains G4-G6 have been deposited with the RCSB 
Protein Data Bank with accession codes: 2FH1, 2FH2, 2FH3 and 2FH4.

\section{Acknowledgements}

We are grateful to the MAX-Lab for the access to their synchrotron radiation facility. For financial support, R.C.R. thanks the Swedish Medical Science Research Council, the Swedish Natural Science Research Council, the EMBO YIP scheme and A*STAR, and L.D.B. thanks the Heart and Stroke Foundation of British Columbia and the Yukon and the Canadian Institutes for Health Research.

\section{References}

1. Allen, P. G. (2003). Actin filament uncapping localizes to ruffling lamellae and rocketing vesicles. Nature Cell. Biol. 5, 972-979.

2. Hartwig, J. H. (1992). Mechanisms of actin rearrangements mediating platelet activation. J. Cell. Biol. 118, 1421-1442.

3. Larson, L., Arnaudeau, S., Gibson, B., Li, W., Krause, R., Hao, B. H. et al. (2005). Gelsolin mediates calciumdependent disassembly of Listeria actin. Proc. Natl Acad. Sci. USA, 102, 1921-1926.

4. Robinson, R. C., Mejillano, M., Le, V. P., Burtnick, L. D., Yin, H. L. \& Choe, S. (1999). Domain movement in gelsolin: a calcium-activated switch. Science, 286, 1939-1942.

5. Burtnick, L. D., Urosev, D., Irobi, E., Narayan, K. \& Robinson, R. C. (2004). Structure of the N-terminal half of gelsolin bound to actin: roles in severing, apoptosis and FAF. EMBO J. 23, 2713-2722.

6. Burtnick, L. D., Koepf, E. K., Grimes, J., Jones, E. Y., Stuart, D. I., McLaughlin, P. J. \& Robinson, R. C. (1997). The crystal structure of plasma gelsolin: implications for actin severing, capping, and nucleation. Cell, 90, 661-670.

7. Lamb, J. A., Allen, P. G., Tuan, B. Y. \& Janmey, P. A. (1993). Modulation of gelsolin function-activation at low $\mathrm{pH}$ overrides $\mathrm{Ca}^{2+}$ requirement. J. Biol. Chem. 268, 8999-9004.

8. Kothakota, S., Azuma, T., Reinhard, C., Klippel, A., Tang, J., Chu, K. T. et al. (1997). Caspase-3-generated fragment of gelsolin: effector of morphological change in apoptosis. Science, 278, 294-298.

9. Pope, B., Way, M. \& Weeds, A. G. (1991). Two of the three actin-binding domains of gelsolin bind to the same subdomain of actin-implications for capping and severing mechanisms. FEBS Letters, 280, 70-74.

10. Selden, L. A., Kinosian, H. J., Newman, J., Lincoln, B., Hurwitz, C., Gershman, L. C. \& Estes, J. E. (1998). Severing of F-Actin by the amino-terminal half of gelsolin suggests internal cooperativity in gelsolin. Biophys. J. 75, 3092-3100.
11. Weeds, A. G., Gooch, J., McLaughlin, P., Pope, B., Bengtsdotter, M. \& Karlsson, R. (1995). Identification of the trapped calcium in the gelsolin segment 1-actin complex-implications for the role of calcium in the control of gelsolin activity. FEBS Letters, 360, 227-230.

12. Pope, B., Maciver, S. \& Weeds, A. (1995). Localization of the calcium-sensitive actin monomer binding-site in gelsolin to segment- 4 and identification of calciumbinding sites. Biochemistry, 34, 1583-1588.

13. Khaitlina, S., Walloscheck, M. \& Hinssen, H. (2004). Calcium-induced conformational changes in the C-terminal half of gelsolin stabilize its interaction with the actin monomer. Biochemistry, 43, 12838-12845.

14. Kiselar, J. G., Janmey, P. A., Almo, S. C. \& Chance, M. R. (2003). Visualizing the $\mathrm{Ca}^{2+}$-dependent activation of gelsolin by using synchrotron footprinting. Proc. Natl Acad. Sci. USA, 100, 3942-3947.

15. Choe, H., Burtnick, L. D., Mejillano, M., Yin, H. L., Robinson, R. C. \& Choe, S. (2002). The calcium activation of gelsolin: insights from the $3 \AA$ structure of the G4-G6/actin complex. J. Mol. Biol. 324, 691-702.

16. Kolappan, S. L., Gooch, J. T., Weeds, A. G. \& McLaughlin, P. J. (2003). Gelsolin domains 4-6 in active, actin-free conformation identifies sites of regulatory calcium ions. J. Mol. Biol. 329, 85-92.

17. Narayan, K., Chumnarnsilpa, S., Choe, H., Irobi, E., Urosev, D., Lindberg, U. et al. (2003). Activation in isolation: exposure of the actin-binding site in the C-terminal half of gelsolin does not require actin. FEBS Letters, 552, 82-85.

18. McLaughlin, P. J., Gooch, J. T., Mannherz, H. G. \& Weeds, A. G. (1993). Structure of gelsolin segment-1actin complex and the mechanism of filament severing. Nature, 364, 685-692.

19. Kazmirski, S. L., Isaacson, R. L., An, C., Buckle, A., Johnson, C. M., Daggett, V. \& Fersht, A. R. (2002). Loss of a metal-binding site in gelsolin leads to familial amyloidosis-Finnish type. Nature Struct. Biol. 9, 112-116.

20. Evans, C. H. (1983). Interesting and useful biochemical properties of lanthanides. Trends Biochem. Sci. 8, 445-449.

21. Collaborative Computing Project Number 4. (1994). The CCP4 suite: programs for protein crystallography. Acta Crystallog. sect. D, 50, 760-763.

22. Jones, T. A., Zou, J. Y., Cowan, S. W. \& Kjeldgaard, M. (1991). Improved methods for building protein models in electron-density maps and the location of errors in these models. Acta Crystallog. sect. A, 47, 110-119.

23. Merritt, E. A. \& Murphy, M. E. P. (1994). Raster3D version-2.0-a program for photorealistic molecular graphics. Acta Crystallog. sect. D, 50, 869-873.

24. Esnouf, R. M. (1999). Further additions to MolScript version 1.4, including reading and contouring of electron-density maps. Acta Crystallog. sect. D, 55, 938-940. 